\title{
"Mejor que los autóctonos". El capital social y su manifestación en el rendimiento escolar de los jóvenes de origen filipino en Barcelona
}

\section{"Better than the locals". Social capital and its display in the academic success of young Filipinos in Barcelona}

\author{
Eva MARXEN \\ Universitat Rovira i Virgili, Tarragona
}

Recibido: Julio 2012

Aceptado: Diciembre 2012

\section{Resumen}

Muchos jóvenes de origen filipino son reconocidos en Barcelona por su elevado rendimiento académico y por la escasa conflictividad social que generan. En el presente trabajo examinaremos las causas y las consecuencias de todo ello y analizaremos algunos de los factores cruciales que caracterizan a este colectivo: el capital humano y social, así como la circulación transcultural de la educación como valor clave de las familias.

El estudio es el resultado de una etnografía desarrollada durante cinco años en Barcelona y Filipinas.

Palabras clave: Capital social, capital humano, escolarización selectiva, asimilación segmentada, aculturación selectiva.

\begin{abstract}
Many youngsters of Filipino origin are rewarded in Barcelona because of their academic success and low social conflictivity. This paper discusses the reasons and consequences and analyzes some of the crucial factors which characterize the Filipino community: its human and social capital, as well as the transcultural circulation of education as a key value in the families. This study is the result of a five-year ethnography between Barcelona and the Philippines.
\end{abstract}

Keywords: Social capital, human capital, selective schooling, segmented assimilation, selective acculturation.

En el presente trabajo aportamos conocimiento sobre los procesos de escolarización, aprovechamiento de capital humano, acumulación de capital social y estrategias de integración educativa y social de jóvenes de origen filipino en Barcelona. La comunidad filipina destaca con el despliegue de un sofisticado capital social: ofrece a los recién llegados una recepción y una orientación óptimas, así como apoyo para 
resolver los distintos asuntos inherentes al proceso migratorio, ayuda para combatir el sufrimiento derivado de su circunstancia social y hacer frente a la explotación laboral. Simultáneamente, la comunidad requiere de sus miembros una dedicación comprometida en forma de trabajos voluntarios.

La eficacia del capital social se manifiesta por los cuidados ofrecidos por la comunidad y, especialmente, en los asuntos concernientes a la educación de los jóvenes, cuya importancia circula a nivel transnacional y con la que se aspira a conseguir un ascenso social.

\section{Breve descripción metodológica}

Para analizar las realidades inmigratorias, se considera crucial incluir las dinámicas emigratorias, así como los flujos y las prácticas transnacionales. En este sentido, el presente trabajo es el resultado de una etnografía multisituada realizada entre los años 2007 y 2011 en Barcelona y Filipinas (con una estancia en el Population Institute, University of the Philippines, Diliman, Manila; y en las provincias de Batangas y Laguna). En ambos lugares hemos realizado observaciones participativas, análisis documental y hemos entrevistado a estudiantes de institutos de enseñanza secundaria y de primer ciclo universitario, así como a padres, madres y profesionales que trabajan con los jóvenes. En Filipinas hemos conocido a muchos académicos (antropólogos, sociólogos, demógrafos, psicólogos) y en Barcelona a los actores clave de la comunidad filipina y a personas procedentes de otros lugares que trabajan, conviven o estudian con gente de origen filipino. También, se han realizado talleres con la modalidad de la etnografía desde el arte (véase sobre el funcionamiento: Marxen, 2009) y desde 2005 hasta la actualidad vivimos en el Raval, en medio de la concentración demográfica filipina. Además, hemos llevado a cabo dos seguimientos de familias transnacionales entre los dos continentes. En total, hemos trabajado con 45 informantes en Filipinas y 124 en Barcelona.

\section{El contexto sociopolítico de los jóvenes: las migraciones filipinas y la comunidad filipina en El Raval}

La característica principal de Filipinas como emisor de emigrantes radica, dejando aparte los aspectos cuantitativos, en el hecho de que los flujos migratorios que genera no se concentran en uno o unos pocos países sino que son diaspóricos, es decir, llegan a casi todo el mundo. La mayoría emigra durante poco tiempo, aunque también hay migrantes permanentes e irregulares (todos son OFW, Overseas Filipino Worker). Trabajan en muchos campos diferentes, tanto en puestos no cualificados como de alta cualificación y especialización, y se han concentrado en las áreas de enfermería, trabajo doméstico y marinería (Asis, 2008).

Diáspora se entiende aquí en un triple sentido: en primer lugar, como una dispersión de los filipinos fuera de su país; en segundo lugar, como causa de una doble relación y doble lealtad: con el país de origen y con el país de destino; y, en tercer lugar, como la cercanía a una comunidad global imaginada de emigrantes filipinos dispersos por el mundo, un transnacionalismo compartido por todas las comunidades filipinas (Parreñas, 1998). Esta triple definición tiene efectos sobre la transnacionalidad y 
transculturalidad, que son conceptos clave para las migraciones filipinas y que tienen sus consecuencias para las prácticas educativas y sus valoraciones.

En el siglo XX empezaron las primeras migraciones organizadas de filipinos, lo que se formalizó con el Manpower Export Program, con el que el dictador Marcos, en el año 1974, declaró oficialmente a Filipinas como productor y exportador de capital humano, siendo éste su papel en la división internacional del trabajo. Se trataba de desarrollar, promover y regular el empleo transnacional. En realidad fue una estrategia para paliar la creciente crisis económica y, en última instancia, sirvió para garantizar el ingreso estable de remesas en forma de divisas.

Después de la destitución de Marcos, en 1986, la emigración planeada fue intensificada por los presidentes posteriores, que introdujeron el concepto propagandístico de "los nuevos héroes y nuevas heroínas de nuestra patria" (Parreñas, 1998: 53). El Estado convierte en objetos a sus ciudadanos, que son considerados generadores de divisas, productos elaborados en Filipinas; se trata de commodified bodies of overseas workers, en el sentido de que el Estado gana plusvalía enviando a los emigrantes por el mundo, beneficiándose de sus remesas y de los negocios creados alrededor del deseo colectivo de emigrar, como, por ejemplo: las múltiples agencias de reclutamiento migratorio (Baggio, 2008; Parreñas, 1998; Zanfrini y Asis, 2006).

Por supuesto, en Filipinas, la gran cantidad de mano de obra emigrada solo esconde los problemas estructurales de la economía (véase Bello, 2004). Las migraciones internacionales no pueden sustituir a las políticas de desarrollo no llevadas a cabo en el país y tampoco son los emigrantes los responsables de sustituirlas y compensarlas, de modo que millones de filipinos siguen emigrando al no ver futuro en su propio país. Como consecuencia, en Filipinas se observa una verdadera cultura de la emigración que corresponde al deseo colectivo de trabajar en el extranjero.

Tanto en Barcelona como a nivel mundial se considera que las comunidades filipinas están "bien adaptadas", incluso mimetizadas, y que no causan problemas sociales (mis observaciones en el Raval; para Italia, Baggio, 2009; y, para Estados Unidos, Le Espiritu y Wolf, 2001). En Barcelona, la comunidad filipina ${ }^{1}$ se empezó a formar a finales de los años setenta. Dicha comunidad se concentra en el Raval, donde están ubicadas tanto las viviendas como las sedes de los diferentes centros, asociaciones e iglesias (la católica y las protestantes) filipinas. El $60 \%$ de la población filipina vive en el noroeste del Raval, en el triangulo formado por las calles Riera Alta, La Cera y Riera Baixa. Sin embargo, no se trata de una zona exclusivamente habitada por filipinos. De hecho, en el Raval ningún colectivo llega a mucho más del $10 \%$ y no hay zonas determinadas para una sola etnia, más bien se caracteriza por una extrema diversidad social (Subirats y Rius, 2006).

\footnotetext{
${ }^{1}$ Se entiende como comunidad filipina el conjunto de personas de origen filipino que se han organizado de manera muy formal en el Raval y que se consideran ellos mismos comunidad: "FilCom" (Filipino Community).
} 
La comunidad filipina cuenta en el Raval con una fuerte red comunitaria propia que Gutiérrez-Otero ha denominado "sistemes de benestar de la comunitat filipina a Barcelona" (2007: 59) y que parecen repetir en Barcelona el modo de vida comunitario de Filipinas -allí debido a la ausencia de prestaciones del Estado. Destaca la confusión entre grupos religiosos y cívicos, así como la fuerte influencia de la Iglesia Católica (en congruencia con Gutiérrez-Otero, 2007). La red de grupos filipinos (la parroquia, el Centro Filipino, la escuela Iskwelang Pinoy, el KALIPI ${ }^{2}$ y la revista Ang Bagong Filipino) funciona como bolsa de trabajo, de vivienda y de contactos, y también como servicio jurídico, de apoyo espiritual y psicológico, así como cuidado en caso de malestares. Por su parte, la Iglesia autóctona, con sus instituciones, ha funcionado desde los inicios de la inmigración filipina como el sostén principal de los recién llegados y ha facilitado el desarrollo de una comunidad formalmente organizada que se ocupa especialmente de la educación de los niños y de los jóvenes. Éste es un aspecto que diferencia claramente el desarrollo de la inmigración filipina respecto al de otras culturas y países no cristianos.

Los actores clave de la comunidad manifiestan la necesidad y la voluntad de integrarse en la sociedad de acogida y de no causar problemas en el lugar de destino. Asimismo, consideran que dicha integración pasa por mantener sus raíces filipinas y, por este motivo, muestran un fuerte deseo de familiarizar a los jóvenes y niños con ellas.

\section{Padres y madres que emigran}

En Filipinas, mucha gente formada y con titulaciones superiores se marcha del país, lo cual conlleva una fuga de capacidades humanas. En este sentido, el hecho de que los padres tengan un alto nivel de formación supone que pueden apoyar y evaluar los progresos educativos de sus hijos. El capital humano, para el que la formación es un factor clave, de muchos adultos filipinos -aunque no de todos- es relativamente alto debido a que cuentan con titulaciones académicas superiores. Emigrando, los padres realizan una inversión con la expectativa de obtener un futuro mejor para sus hijos y para toda la familia que podría conseguirse a través de una buena educación, traducida después en unos ingresos más altos.

Se ha observado que los padres inmigrantes en general tienen grandes expectativas en cuanto a la educación de sus hijos, ya que la consideran como un elemento clave para el ascenso social, y que un alto nivel de educación de los progenitores (sobre todo de las madres) es uno de los factores más decisivos para el éxito escolar de los hijos (por ejemplo: Marí-Klose, Marí-Klose, Granados, Gómez-Granell y Martínez, 2009; Feixa, Romaní, Halim, Latorre Reolon, Porzio y Rodríguez, 2010; Carrasco, Pàmies y Beltran, 2009). Además, también se ha demostrado (Wolf, 1997) que los padres con un

\footnotetext{
${ }^{2}$ Federación de Entidades Cívicas y Religiosas Filipinas en Barcelona que incluye, entre otros, a los líderes religiosos de las iglesias católica y protestante, así como a grupos laicos. Se legalizó como Federación el 18 de noviembre de 2009 e incluye 13 entidades que son miembros y siete que tienen el estatus de colaboradoras.
} 
capital humano alto controlan mejor los avances académicos de sus hijos porque tienen más recursos intelectuales y saben escoger sanciones más significativas para que los hijos les obedezcan. Aparte del nivel formativo de los padres, también es decisivo el grado de implicación en los procesos escolares de sus hijos:

"El grado de implicación de los padres en la educación de sus hijos y la 'calidad' de las orientaciones, habilidades y conocimientos transmitidos tiene efectos decisivos en las trayectorias educativas de los menores. Son lo que se conoce como capital social y capital cultural de las familias" (Marí-Klose et al., 2009: 185). En cuanto al capital social, entendido como la riqueza de las relaciones intra e interfamiliares, veremos que en el caso de la comunidad filipina son decisivas las relaciones comunitarias que facilitan y promueven la educación de los jóvenes. Como capital cultural, en el ámbito educativo se incluyen todos los recursos, no solo los materiales (como la adquisición de bienes culturales como libros, periódicos, etc.) sino también los que conciernen a estímulos cognitivos (como motivaciones, sanciones y la transmisión de habilidades de estudio) que faciliten la adaptación a las exigencias escolares (Marí-Klose et al., 2009). Se ha observado que una alta implicación de los padres puede compensar las desventajas económicas de los mismos (Marí-Klose et al., 2009) y, al mismo tiempo, que un alto grado de capital humano de los padres solo se hace rentable si se combina con una dedicación de los mismos en los procesos educativos de sus hijos (Coleman, 1988).

Muchos emigrantes filipinos trabajan a menudo por debajo de su nivel académico, ya que los trabajos mal pagados en el Norte Global ofrecen aún una mayor estabilidad que muchos trabajos cualificados en el Sur Global (Parreñas, 2001). Como consecuencia, la noción del sacrificio realizado por los padres es omnipresente, lo que intensifica la motivación de éstos para controlar el rendimiento académico de sus hijos.

\section{Rendimiento escolar}

En la comunidad filipina se pone énfasis en la importancia de tener una buena educación, en el deseo de que los jóvenes filipinos asciendan socialmente y consigan el acceso a la universidad. Los profesores catalanes elogian a menudo a sus alumnos filipinos; de hecho, el título del presente artículo se ha extraído de una entrevista con un profesor catalán y representa la opinión de otros profesores entrevistados que también alaban su conducta, su respeto y su disciplina: los consideran muy ordenados y respetuosos hacia los adultos. A esta facilidad de adaptación contribuyen sin duda los conceptos de hiya (vergüenza) y de pakikisama (convivencia, getting along with), que llevan a los filipinos a evitar situaciones que comprometan su reputación (siempre en concordancia con la reputación colectiva) asegurándose al mismo tiempo el aprecio de los demás (Zanfrini y Asis, 2006) y, en el caso de los jóvenes, el de sus profesores, que valoran muy positivamente su correcto proceder:

“- Y lo que se refiere a mi asignatura, en general, estoy contento con los chicos y chicas filipinos que tengo en clase. Primero, porque tienen una actitud muy correcta, son muy trabajadores, son impecables a la hora de tomar apuntes y de presentar los apuntes y el rendimiento académico y los resultados que tienen luego, pues, como la mayoría de sus 
casos depende de sus aptitudes, de la capacidad intelectual específica para las actividades matemáticas. Pero suele haber, y eso es verdad, sobre todo en bachillerato alumnos, suelen ser chicas, por lo menos a mi me ha ocurrido más con chicas, que despuntan, que sobresalen muchísimo en matemáticas. Tienen una formación científica muy buena.

- ¿Cuándo llegan aquí?

- Cuando llegan aquí.

- ¿Cuándo llegan aquí, a Barcelona, al instituto?

- Sí, luego nosotros nos encargamos de estropearlo, pero de los que vienen aquí, suelen ser los mejores de la clase."

(Profesor de matemáticas, IES 1)

La gran mayoría de los alumnos filipinos reagrupados dominan el inglés y algunos, con un nivel de nativo anglosajón, lo conocen incluso mejor que algunos de sus profesores de inglés del instituto:

“- How do the studies go at school?

L/E: Fine.

- Is it difficult to catch up or easy?

E: They are working better in Philippines, right? And if you come here, you will be the highest one, because other children are just going on and they are not studying...

$\mathrm{L}:$ They just want adventures...

(...)

E: ... and she [la profesora de inglés] telling some things that, and I am telling that is wrong, and she is ignoring me because she wanted to know better than the children...

L: That also happened to me once, the English professor, sometimes I helped him...

$\mathrm{E}:$... and then they get angry...

L: ... because it's shameful, she is correcting you...

E/L: [laughing]

$\mathrm{L}:$... for me it's all right.

- And the other classes, the other subjects like maths...

L: ... Like maths? There is no problem.

E: No problem."

(Dos chicas reagrupadas, IES 1 y escuela católica concertada 2) 
A finales del curso 2007/2008, en el IES 1 la única alumna que pasó la selectividad fue una joven filipina que había llegado a Barcelona hacía solo dos años, sin saber ni castellano ni catalán:

“- ¿Has estudiado mucho los últimos años?

- ¡Sí! ¡Mucho, mucho, mucho pero mucho! Al principio me costaba mucho. Estaba llorando, lloré mucho, en casa. He dicho a mi madre que ya no quería estudiar, ya no quería estudiar. Es que no entendía nada, nada, nada, nada, nada. En clase..., estaba muy callada. Y no sé. No sabía qué decirles.

- ¿Y qué te decía tu madre?

- No sé..., que tengo que probar..., a probarlo, primero... Es que los profesores me han dicho antes que tengo, tenía que bajar.

- ¿Qué te han dicho?

- Que bajar, que tenía que dejar los estudios aquí.

- ¿Ah, sí?

- Sí, es que..., que tenía que estudiar primero el castellano y el catalán.

- ¿Y lo hiciste?

- No, he dicho que no, que quería estudiar. Pues eso. Me costaba castellano y catalán pero las otras asignaturas ya estaban bien. Y matemáticas, es que me gusta mucho, y física también [sonríe]."

(Chica de 18 años, ahora estudia ingeniería informática en la Universidad) ${ }^{3}$

Muchos alumnos dicen no tener muchos problemas de orden académico. En su tiempo libre algunos se dedican a actividades extraescolares ${ }^{4}$ como el deporte o el canto clásico, y reciben clases de piano. El director de la Escola de Músics, situada en el Raval, no para de elogiar a sus alumnos filipinos:

“- Y la disciplina ayuda mucho. Tienen estas facilidades y con disciplina, sobresalen mucho más a los de aquí (...)

- ¿El rendimiento musical ya has dicho es óptimo, no?

- Sí, sí, máximo (...) Tienen, yo sinceramente, unas capacidades muy, mucho mejores que las nuestras (...) Sí, simplemente leer. Son muy rápidos, y de memoria, bueno, ya te lo digo,

\footnotetext{
${ }^{3}$ Estas narrativas de los jóvenes contradicen claramente las afirmaciones de Villarroya (2010) y Baggio (2009) según las cuales, a pesar de las preocupaciones de los adultos filipinos, los jóvenes en Barcelona y Madrid se mostrarían indiferentes ante la educación.

${ }^{4}$ La dedicación con asiduidad a actividades extracurriculares se ha observado entre jóvenes filipinos en EE.UU. (Wolf, 1997) y asimismo en Filipinas, sobre todo entre alumnos con padres y madres en el extranjero.
} 
es tocando dos veces ya se lo saben. O tres, sí (...) Aunque se trabaja pero con los catalanes, aunque lo trabajes... no, no, no llegan o les cuesta más. En cambio, los filipinos, rápidamente lo cogen de memoria. Y tienen una memoria muscular muy ágil, es decir, rápidamente les quedan las cosas en la memoria. Y puede ser por esto, porque trabajan más, y también porque tienen menos cosas y se concentran más (...) Y cantan muy bien, por ejemplo, en las musicales que tenemos en las escuelas públicas, los que hacen solos, son filipinos. ¿Por qué? Porque tienen una voz bonita, buena, afinan bien, en cambio los otros es mucho peor. En general, sí, sí. Así de claro.”

Durante los actos oficiales, la comunidad filipina brinda reconocimiento y elogios a sus jóvenes exitosos. En este sentido, ofrecen un reconocimiento público a los recién graduados, diplomados y licenciados, animando a los demás a seguir su ejemplo, y expresan en público (tal como hacen los responsables de la comunidad filipina en las entrevistas) su orgullo por el rendimiento de los jóvenes.

El sacerdote, como actor clave de la comunidad, también resalta la importancia del sueño del ascenso social de los jóvenes filipinos:

"- And also that is one of the dream of the church because I have to accept, I have to accept that when we come here, much of the Filipinos, the only offer to work, we are being offered, is servicio doméstico... I do not say it is bad...

- ...no, no...

- ...because it is a very noble...

- ...of course. No, I don't say that either but maybe the young people could go...

- ...ya, could go to different, different levels (...) So, once they have that kind of combination [se refiere a la combinación del dominio del idioma y de las formaciones necesarias], maybe, you will see in the hospitals doctors, Filipino doctors, you will see in the Ayuntamiento, maybe [se ríe], in the... Social Services Filipinos (...) that is our dream also, that is our dream."

Las metas de los actores clave de la comunidad filipina apuntan claramente a una integración y a un ascenso en la sociedad catalana, aceptando los que ellos consideran altos y medios cargos laborales como parámetros propios. Sus normas coinciden con las de la sociedad de acogida: terminar una formación o unos estudios académicos para tener un futuro profesional, y constituyen de este modo una forma eficaz de capital social -en el sentido en que lo utiliza Coleman (1988).

Aunque los jóvenes reagrupados tienen desventajas lingüísticas en comparación con los nacidos en Cataluña, saben compensar esta falta con su rendimiento en inglés, matemáticas, ciencias naturales y música; en este sentido llama la atención que los jóvenes que llegan a cursar estudios universitarios optan a menudo por asignaturas en 
las que el idioma ocupa un lugar secundario: ingeniería informática, música ${ }^{5}$, etc. (en congruencia con estudios en EE.UU. con estudiantes de origen asiático que también solían evitar materias que requieren habilidades verbales y lingüísticas: Hsia, 1988; Hune y Chan, 1997). En este contexto conviene considerar que tanto en Filipinas como en la diáspora la educación es considerada en primer lugar como un instrumento para el ascenso social y la integración socioeconómica. La dedicación vocacional a los estudios es considerada un lujo o simplemente no es considerada ni comprendida.

Así pues, hay jóvenes con rendimientos sobresalientes que han llegado relativamente tarde a Barcelona, por ejemplo entre los 13 y los 15 años (véanse las entrevistas arriba), de manera que, en el caso de los jóvenes filipinos, no se cumple la suposición de que los beneficiarios de las experiencias inclusivas e integrativas son aquellos que han llegado al lugar de destino a una temprana edad (Feixa et al., 2010) ${ }^{6}$. Más bien conviene resumir y resaltar que en general "la incorporación tardía a un nuevo sistema educativo condiciona las trayectorias del alumnado, pero que no hipoteca la posibilidad de acabar con éxito la ESO ni la transición a los estudios postobligatorios" (Serra y Palaudàrias, 2010: 146).

Se espera que una buena educación proporcione a los jóvenes un ascenso social, ya sea en Filipinas o en el país de destino. Para poder pagarla, los padres emigran y trabajan a menudo por debajo de su nivel formativo; lo cual suele ser utilizado como argumento de presión sobre sus hijos. Muchos jóvenes sienten y expresan su gratitud y, con su éxito académico, a menudo quieren hacer felices a sus padres y devolverles, "repagar" sus esfuerzos (Zanfrini y Asis, 2006 y Añonuevo y Añonuevo, 2002). En este sentido, podemos ver cómo han interiorizado que el proyecto migratorio familiar tiene como objetivo el éxito educativo y, de este modo, se consideran "deudores de la continuidad del proyecto migratorio familiar" (Pàmies, 2011). A veces, esto les lleva a situaciones de estrés, de sobrecarga, así como a tener sentimientos de culpa y de responsabilidad, sobre todo si les recriminan que no sepan valorar los sacrificios de los padres y de las madres:

“- La presión, la presión es tremenda, pero muy grande, muy grande. La madre llorando, delante de la hija y diciendo, todo esto lo hago por ti, yo estoy sufriendo mucho, estoy trabajando todo el día. (...)

\footnotetext{
${ }^{5}$ En Filipinas he podido observar que se valoran mucho las aptitudes para la música, el canto, la danza, las coreografías y el karaoke. Forman parte de las fiestas y de las reuniones y las respectivas capacidades otorgan mucho reconocimiento social. En este sentido, niños y jóvenes son animados desde muy pequeños a realizar actividades musicales.

${ }^{6}$ Lo mismo ocurre en el lado opuesto en el caso de algunos alumnos de origen marroquí que, a pesar de una temprana escolarización en Cataluña, se encuentran en una situación de desventaja educativa o, incluso, de empeoramiento (Pàmies, 2011). En EE.UU. incluso se ha observado que a mayor tiempo de permanencia más descenso del rendimiento escolar hay (Suárez Orozco y Súarez Orozco, 2008; Portes y Rumbaut, 2001a y 2001b).
} 
Yo creo el discurso generalizado es: yo sufro y trabajo por ti. Y tú deberías, he venido aquí para que estudies. (...)

Y lloran y todo, lloran. Lloran en muchos casos que, pero es que mi madre, yo lo hago por mi madre (...) Para dar, para que mi madre sea feliz, mi madre no sé que, mi madre, o sea para mi madre o para mis padres."

(Profesora de castellano, IES 1; véase también Parreñas, 2006; Wolf, 1997)

En este sentido, el director de la Escola de Músics afirma:

“- Sí. ¡Oh, sus padres! Porque los padres están muy detrás, muy, muy, muy. Son muy, muy, muy controladores, muy duros, muy duros."

Portes y Rumbaut (2001a: 215, 224) hablan de un "strong achievement strive" (un fuerte afán al éxito), que ocasionalmente lleva a presiones extraordinarias de padres a hijos, entre las familias asiáticas de Estados Unidos, incluyendo a los filipinos. Asimismo, Wolf (1997) observó durante su estudio realizado en colleges y escuelas secundarias en California graves alteraciones psíquicas, sobre todo entre las chicas de origen filipino. En aquel estudio los profesores y counselors resaltaban la presión académica extrema por parte de los padres. Sin embargo, los y las jóvenes casi nunca se rebelaron en contra de ellos ya que la expresión abierta de sentimientos negativos contra los padres en Filipinas está extremadamente mal vista. Al mismo tiempo, los chicos y las chicas tampoco acudían a los psicólogos o counselors de los servicios de salud mental o de las escuelas y universidades (coincide con Suzuki, 2002). Esto se debe en gran medida a la vergüenza, hiya, que se asocia con el hecho de recurrir a servicios de salud mental, ya que, según los adultos filipinos, los problemas familiares no deben salir de casa. En las transcripciones de los comentarios de los jóvenes acerca de este tema, aparece cuatro veces en una página la palabra shame (Wolf, 1997: 470). Por el miedo a los rumores, el gossip, entendido como una forma de sanción eficaz de la comunidad para mantenerla hermética, los jóvenes a menudo tampoco se atreven a compartir su malestar con otros familiares o miembros de la comunidad. Por su parte, muchos jóvenes con tendencias suicidas se encuentran encerrados en una situación de soledad, desesperación e ideas autodestructivas. En estos casos las familias se presentan como fuente de conflictos y se percibe un contraste profundo entre la ideología familiar filipina, con la idealización de la cercanía y de los lazos familiares, y la realidad de las prácticas familiares o parentales, que ejercen una presión importante sobre los jóvenes. Tanto en Filipinas como en Barcelona hemos podido comprobar esta discrepancia entre el concepto de familia filipina, que se presenta como una institución idealizada, y una realidad que se aleja mucho de dicho ideal. Hemos observado graves tensiones en las relaciones familiares, sobre todo por temas de presión académica y de exigencias de índole moral por parte de los padres y de las madres, así como de la familia extensa en interrelación con la comunidad. Sin embargo, los mismos jóvenes afectados siguen idealizando a sus familias y a la familia en general como institución.

En este sentido, se aboga una vez más por una visión crítica de las minorías modélicas (Wong, Faith Lai, Nagasawa y Lin, 1998) que se caracterizan en términos de 
éxitos académicos de los hijos, un alto nivel de ingresos de los adultos ${ }^{7}$, una baja tasa de criminalidad, pocos problemas articulados de salud mental y, como consecuencia de todo, una tendencia clara a una asimilación no conflictiva y a ascensos sociales (Wong et al., 1998: 1). También se definen por presentar familias unidas y lazos familiares fuertes que brindan apoyo a los jóvenes. Sin embargo, conviene examinar las prácticas en las familias, y si estas son lugares de ligadura y vínculos afectivos o bien de conflictos intensos (Wolf, 1997 y Rumbaut, 1997).

\section{Circulación transnacional}

Los jóvenes señalan con unanimidad la importancia de la influencia parental en sus rendimientos académicos. También mencionan, tanto los jóvenes como los adultos, la importancia y el alto valor que tiene la educación en Filipinas. En este sentido, la directora del Centro Filipino me indicó que en Filipinas los padres consideran la educación como una herencia para sus hijos, a pesar de (o a causa de) no poder dejarles dinero o bienes. Los padres también consideran que la educación es la mejor inversión ya que, al contrario de lo que ocurre con el dinero, no se puede gastar o desgastar (también Zanfrini y Asis, 2006).

La alta valoración de la educación, en combinación con la disciplina y el rigor, se extienden a nivel transcultural entre la comunidad filipina en Barcelona, otros lugares de la diáspora y Filipinas. Si tomamos como unidad de análisis a los alumnos de secundaria y a los estudiantes universitarios entrevistados en Barcelona y en Filipinas (en la zona metropolitana de Manila y en las provincias de Batangas y Laguna, en el sur de Luzon), veremos que se puede subdividir a los jóvenes en los siguientes grupos:

- Los reagrupados en Barcelona, a veces nacidos aquí, enviados allí y ahora otra vez reagrupados aquí, que han ido a la escuela en Filipinas y conocen muy bien la disciplina y el rigor. A menudo consideran inferior la educación de las escuelas de Barcelona.

- Los que han sido reagrupados en Barcelona después de haber vivido en Filipinas y en otros países antes de llegar a Barcelona: han ido a la escuela allí y conocen muy bien la disciplina y el rigor. A menudo consideran inferior la educación de las escuelas de Barcelona. Hablan diferentes idiomas debido a sus estancias en distintos países, por ejemplo: inglés, tagalo, alemán, luxemburgués...

- Los que han nacido y han vivido siempre aquí pero han visitado las escuelas de Filipinas, como oyentes, durante las vacaciones. Allí han conocido la disciplina y el rigor.

- Los que han nacido, han vivido y han sido escolarizados siempre en Barcelona pero que por falta de disciplina y obediencia o por haber tenido relaciones

\footnotetext{
${ }^{7}$ Se ha comprobado en EE.UU. que los relativos altos ingresos de las familias de origen asiático se deben a menudo, entre otras cosas, al mayor número de miembros familiares que contribuyen a dichos ingresos. Finalmente, el ingreso anual per cápita de los americano-asiáticos estaba claramente por debajo del de los "blancos" (Suzuki, 2002).
} 
sexuales prematuras son enviados a Filipinas para disciplinarlos. Ahora viven y van a la escuela allí y, por tanto, conocen muy bien la disciplina y el rigor de allí.

- Los que han sido reagrupados aquí y conocen muy bien la disciplina y el rigor de Filipinas. Ahora se les amenaza con enviarles de nuevo allí para disciplinarles.

- Los que siempre han vivido y estudiado en Filipinas. Tienen padres y/o madres en el extranjero y conocen solo las escuelas de allí, que sus padres y madres pagan con sus remesas.

En todos los casos los padres y las madres siguen muy de cerca los estudios y los controlan, ya sea in situ o desde el extranjero, a través de medios de comunicación como el teléfono, los mensajes de texto o Internet, o durante los viajes de encuentro. También, en todos los casos, hay hermanos y/o primos en Barcelona y/o en el extranjero o en Filipinas que escuchan y reproducen los discursos de la disciplina, el rigor y la importancia de la educación. De este modo, el mensaje circula entre todos los afectados en todos los países, incluyendo a los profesores de los lugares de destino de la diáspora filipina y a los investigadores filipinos e internacionales (Aguilar, 2009; Parreñas, 1998 y 2006; Zanfrini y Asis, 2006). Su circulación y su transmisión constante y reiterada entre Filipinas y los distintos países de la diáspora filipina confirman una vez más las prácticas transnacionales de los filipinos.

Otro resultado de las trayectorias escolares transnacionales se refleja en una situación aventajada de estos alumnos debida a los sólidos conocimientos que adquieren en las escuelas filipinas en disciplinas como las matemáticas y las ciencias naturales, así como el dominio que tienen del inglés y, en algunos casos, de otros idiomas. En este contexto se ha hablado de "ventajas premigratorias" y "contextos de salida" (Suárez Orozco y Suárez Orozco, 2008; y Dronkers, 2010, quien sitúa la calidad de la educación en el país de origen encima de todos los otros factores, menos los de orden individual). Una buena educación en los lugares de origen sería crucial por la capacidad facilitadora de los padres como también por la transmisión de la implicación conductual y los habitus de aprendizaje.

\section{La escolarización selectiva}

En general, parece que los principales agentes de socialización y de control social de los jóvenes filipinos afincados en Barcelona (las escuelas, la familia y toda la red comunitaria filipina del Raval con la parroquia, el Centro Filipino y la Escuela Pinoy) coinciden en valores como el rendimiento académico, la fe católica y la incorporación exitosa en el mundo laboral. En este contexto, el instituto público no transmite oficialmente la educación católica y por ello cuenta cada vez con menos alumnos de origen filipino, ya que a menudo son enviados a las escuelas privadas. Así pues, una diferencia importante entre los diferentes grupos de inmigrantes se manifiesta a la hora de escoger escuela: la comunidad filipina prefiere claramente los centros católicos 
concertados, mientras que por ejemplo un $81.2 \%$ de los niños y jóvenes latinoamericanos y un $89.8 \%$ de los africanos acuden a las escuelas públicas (Feixa et al., 2010; en cuanto a la selección de colegios privados por parte de las familias filipinas mi investigación cualitativa coincide con la cuantitativa de Aparicio, Portes y Haller, 2009).

En el Raval, en algunas escuelas católicas la mitad del alumnado es filipino y en un colegio católico algunas clases tienen entre un 90 y un 100\% de alumnos filipinos, mientras que en los últimos años en el instituto público situado al lado casi ya no hay nuevas inscripciones de alumnos de origen filipino. Por ejemplo, en octubre de 2008 hubo únicamente una, mientras que en años anteriores había habido muchas más. En aquel instituto es un hecho que cada año muy pocos alumnos (entre 1 y 3 ) llegan a presentarse a la selectividad. En este punto los jóvenes de origen filipino se distinguen una vez más de otros jóvenes de origen extranjero, especialmente los que se encuentran con sus familias en situaciones socioeconómicas desfavorecidas, ya que estos últimos suelen matricularse en escuelas de bajo prestigio donde "no están expuestos a inputs que les permitan mejorar su situación social, ni escolar y ven limitadas sus expectativas" (Pàmies, 2011, refiriéndose a Suárez-Orozco, Suárez-Orozco y Todorova, 2008, así como a Gándara y Contreras, 2010).

Por otro lado, Aparicio et al. (2009) han observado que los padres y sobre todo las madres inmigrantes de los alumnos matriculados en los colegios concertados en Barcelona tienen un nivel educativo significativamente más alto en comparación con los padres de los alumnos que acuden a las escuelas públicas. Esto se refleja también en las aspiraciones y expectativas que tienen los alumnos sobre su futuro: en los colegios concertados son claramente más altos. Se ha observado que el rendimiento escolar de los estudiantes no depende sólo de los contextos familiares, sino también de las características de los centros escolares, donde son decisivas la composición social del alumnado y de sus familias (Marí-Klose et al., 2009). Ahora bien, las escuelas públicas han sido consideradas unas de las instituciones cruciales de aculturación de los jóvenes (Portes y Rumbaut, 2001a). La comunidad filipina, cuyos adultos se quejan, igual que los padres y las madres de otros países ${ }^{8}$, de la permisividad de la educación local y critican la laxitud moral y académica de los centros públicos, parece escoger adrede escuelas privadas donde se transmite la fe católica, ciertos valores morales y en las que más de la mitad de los alumnos proceden de Filipinas. Los adultos no suelen valorar los posibles efectos asimiladores de las escuelas públicas catalanas y, sobre todo, ven en la ausencia de la transmisión de la moral católica así como del rigor académico un riesgo que puede debilitar la cohesión interna de la comunidad. En la comunidad se transmite la información acerca de dónde es mejor matricular a los hijos y estos flujos de información representan otra forma de su capital social (Coleman,

\footnotetext{
${ }^{8}$ Véanse por ejemplo Carrasco et al., 2009. También es una crítica reiterada que he escuchado como conductora de grupos de mujeres inmigrantes: critican mucho la permisividad de los maestros y profesores locales. Sin embargo, Aparicio et al. (2009: 25) señalan que la gran mayoría de los alumnos de $2^{\circ}$ y $3^{\circ}$ de ESO de su investigación valora "positivamente la actitud de los profesores, la disciplina y la calidad de la enseñanza".
} 
1988). La concentración de alumnos de origen filipino en unas pocas escuelas privadas católicas puede maximizar la cohesión interna de la comunidad que, en contra de las propuestas de distribución y dispersión del alumnado extranjero entre diferentes escuelas y barrios, puede "empoderar a la minoría desde el reconocimiento de su agencia" (Carrasco et al., 2009: 22) generando redes de apoyo ${ }^{9}$. Conjuntamente con el apoyo de la comunidad y la Iskwelang Pinoy, donde los sábados por la tarde a los niños y jóvenes les enseñan tagalo, inglés y filipiniana -valores e historia de Filipinas-, se pueden "crear auténticas comunidades de aprendizaje en que diversos agentes comunitarios (...) se impliquen en la creación de entornos educativos propicios para el logro educativo" (Marí-Klose et al., 2009: 178, 179).

Todos los grupos que forman la comunidad filipina están convencidos de la necesidad de preservar la propia cultura y, por tanto, la transmiten a los jóvenes en la Iskwelang Pinoy y en otros actos y actividades de la comunidad. Con estas iniciativas, la comunidad parece querer amortiguar las posibles influencias negativas de la sociedad de acogida. Los escenarios en los que ubican dichas influencias son las escuelas públicas y, sobre todo, algunas calles del Raval, donde viven la mayoría de ellos y donde temen que los jóvenes tengan contacto con la prostitución y la drogadicción. Ahora bien, la comunidad filipina quiere evitar una asimilación descendente en el lugar de destino y la consecuente exclusión social de sus hijos (Portes y Rumbaut, 2001a).

En Filipinas, pude observar personalmente cómo se hace una clara diferenciación entre las necesidades que requieren la educación de los niños y la de los adolescentes. Los últimos son más sospechosos de desviaciones como el consumo de drogas o la frecuentación de malas compañías y requieren la intermediación de guías, mecanismos de control y figuras paternales y maternales de autoridad, realidad que también me confirmaron los informantes adultos filipinos en Barcelona (en congruencia con Aguilar, 2009 y Pingol, 2007). Las chicas, en este sentido, necesitan aún más control que los chicos, especialmente por lo que ellos consideran desviaciones sexuales.

Aunque en general en Filipinas las relaciones entre ambos sexos están muy reglamentadas, cada familia inmigrada toma sus decisiones al respecto. Por la supuesta vulnerabilidad de los y las adolescentes, algunos optan por no reagruparlos en Cataluña, sobre todo si confían en los padres sustitutos. En caso contrario, algunos prefieren regresar al lugar de origen para vigilar personalmente a los y las adolescentes (Aguilar, 2009), mientras que otros inician el proceso de reagrupación en el lugar de

\footnotetext{
${ }^{9}$ Al mismo tiempo, el abandono de los institutos públicos por parte de las familias filipinas con su capital humano pone de nuevo de manifiesto que los centros públicos de poco prestigio empeoran precisamente porque los ciudadanos con mayor capital humano, social y cultural conocen sus deficiencias y no quieren frecuentarlos. Les restan inputs positivos, les despojan de capital social y privan a los alumnos de estas escuelas de oportunidades de progreso académico (Marí-Klose et al., 2009).
} 
destino y otros consideran la posibilidad de enviarlos de vuelta, sobre todo si ya los ven "perdidos" en la sociedad de acogida".

Tanto en Filipinas como en la diáspora se incluye entre los hijos "fracasados" a los que no terminan la escuela, a los que se enganchan a las drogas y a las malas compañías, a las chicas que se quedan embarazadas antes del matrimonio y antes de terminar su formación académica o que contratan matrimonios prematuros (en congruencia con Parreñas, 1998, así como Zanfrini y Asis, 2006). En definitiva, esta categoría abarca a todos los que no poseen bait, que significa "consideración para los demás, madurez y conductas socialmente aceptadas". Un niño aún no debe tener baít pero, un adulto, sí (Aguilar, 2009: 229-230).

En Barcelona sobre todo, el asunto Crissanto, el caso de un joven de origen filipino drogado que supuestamente quemó a un indigente, ha traumatizado y movilizado a la comunidad. Desde entonces se preocupan enormemente por el tiempo libre de los jóvenes y tratan de evitar que se queden en las calles, desocupados, y sobre todo que se repita un episodio de estas características. Para lograrlo están permanentemente desarrollando "estrategias que pasan por formalizar los canales de apoyo y participación intracomunitaria, desde el asociacionismo hasta la observancia religiosa". La comunidad cumple funciones familiares, con el objetivo de "que un mayor control sobre los hijos e hijas favorezca el éxito académico y la integración social a través de la mejora laboral y económica, al apartarlos de prácticas juzgadas como no deseables (...) Así se fortalezcan las pautas culturales de cohesión y supervisión comunitaria" (Carrasco et al., 2009: 22-23, que han observado prácticas parecidas entre familias marroquíes de su estudio).

Hay también algunos jóvenes de familias filipinas que no tienen tanto éxito escolar como otros de sus compatriotas. Algunos profesionales y jóvenes relatan casos de alumnos que terminan la escuela después de $4^{\circ}$ de ESO para empezar a trabajar en la restauración, sin aspirar a una formación profesional ni al bachillerato. Las causas más comunes son los problemas lingüísticos o el deseo de ganar dinero lo antes posible. A veces hay familias que se encuentran en una situación de bifurcación respecto a sus hijos: los padres, aparte de tener en cuenta los aspectos legales para lograr la reagrupación, también se guían por las oportunidades laborales que van a tener los reagrupados para contribuir a los ingresos familiares. Al mismo tiempo, los hijos residentes en Filipinas se benefician de las posibilidades educativas financiadas con las remesas (Zanfrini y Asis, 2006).

Otros jóvenes filipinos llegan de Filipinas con una formación escasa y sin afán por seguir formándose. Esta tendencia se debe a la decepción que les sobreviene cuando ven que, tanto en Filipinas como en el extranjero, sus familiares u otros compatriotas con estudios superiores tienen que ejercer trabajos inferiores a su cualificación. Decididamente no quieren llegar al college o apenas terminan la escuela obligatoria porque ven que esto no les garantizará un buen empleo con un sueldo digno que les

\footnotetext{
${ }^{10}$ Se trata de una práctica que no se limita a los filipinos ni a los residentes en Cataluña: véase Portes y Rumbaut, 2001a, sobre familias dominicanas en EE.UU.
} 
permita mantener a una familia. Al mismo tiempo, piensan que pueden empezar directamente en el extranjero como trabajadores domésticos, ya que estas actividades no requieren una larga formación, y de ahí que opten por ahorrársela y sigan a sus familiares y vecinos a España, Italia, Hong Kong, etc. (Añonuevo y Añonuevo, 2002; Scalabrini Migration Center, 2006; Zanfrini y Asis, 2006). La comunidad filipina en Barcelona es consciente de esta realidad y quiere tomar medidas:

“- ¿Hay más personas, en la comunidad filipina, que también, como tú, están preocupados por el fracaso escolar de los jóvenes?

- Sí, sí. Yo soy también, estoy montando un... estoy montando un grupo, de las madres aquí, para que podemos hacer algo. A los que tienen títulos universitarios, por ejemplo, como una madre como yo, porque título de, tengo título, soy profesora de ESO en Filipinas, por eso tengo mucho cariño con estos jóvenes. Y estoy montando un grupo con ocho señoras filipinas que también tiene titulación y también quiere, es que queremos montar esta asociación para dedicar a estos jóvenes.

- ¿Ayudar académicamente?

- Ayudar.

Tenemos reunión para el día 25 de este mes y a ver, ¿qué podemos hacer? Claro, vamos a necesitar también el apoyo del consulado, de parroquia (...) Y que tenemos que, es que tenemos que salvar, ¿sabes? Es muy triste ver los jóvenes así."

(Actora clave de la comunidad y madre de dos hijos adolescentes)

\section{Asimilación segmentada y aculturación selectiva}

Según el concepto de la asimilación segmentada de Portes y Rumbaut (2001a y 2001b), las trayectorias migratorias varían significativamente por una serie de factores, entre los cuales destacan cuatro que se pueden considerar como decisivos:

1) La historia de la primera generación del colectivo, que incluye factores de capital humano, la composición y unidad familiar, así como el modo de recepción en la sociedad de acogida (por ejemplo, en el ámbito legal).

2) La aculturación entre padres e hijos según las integraciones normativas.

3) Las barreras culturales y económicas que afronta la segunda generación en su lucha por lograr una adaptación exitosa.

4) Los recursos familiares y de la comunidad para afrontar estas barreras.

A continuación, desarrollaremos cada uno de los cuatro apartados:

1. Como ya se ha explicado anteriormente, el capital humano de muchos adultos de origen filipino es alto. 
Respecto a la situación legal de los filipinos en Barcelona, al tratarse de individuos que proceden de un país que era una colonia española, existe la posibilidad de solicitar la nacionalidad después de dos años de residencia legal. Como obstáculo cabe reseñar que no pocos adultos se encuentran con dificultades a la hora de querer homologar sus títulos académicos.

Además, la legislación de España prevé la posibilidad de la reagrupación familiar y muchas familias de origen filipino se han reagrupado en Barcelona. Estos trámites a menudo se han realizado después de una trayectoria como familia transcultural que ha supuesto separaciones y vicisitudes que perjudican frecuentemente las relaciones entre padres, madres e hijos. La familia, como institución, es clave en Filipinas y la cercanía entre los miembros familiares, sus ayudas mutuas y sus reuniones permanentes son considerados por los propios filipinos como factores centrales que definen el ser filipino (Wolf, 1997). Sin embargo, también las familias filipinas pueden ser focos de conflictos (véase arriba). Además, se han observado conflictos y transgresiones como los casos de mujeres para las que la emigración significa una solución para poderse separar de sus maridos (Ribas, 1994 y 2004/2005; Juliano, 2003; Parreñas, 1998 y 2006). También se han observado casos de hijos reagrupados que se enteran a su llegada de que sus padres o madres tienen una nueva pareja (profesoras de la escuela católica concertada 1). Sin embargo, los posibles conflictos alrededor de los procesos de reagrupación parecen no conllevar rebeliones y críticas en público.

La comunidad filipina está formalmente organizada y la recepción comunal resulta sumamente eficaz. En general, se caracteriza por un alto capital social. A pesar de estas ayudas, las redes que se han establecido pueden implicar el riesgo de concentrarse solo en los trabajos de servicios para los que los filipinos, y sobre todo las filipinas, gozan de buena reputación debido a su nivel formativo, dominio del inglés, fiabilidad y diligencia $y$, por este motivo, canalizan así un capital humano considerable en ocupaciones inferiores. En Barcelona se ha observado que algunos jóvenes llegan con la expectativa de poder insertarse a través de la comunidad en el servicio doméstico o de hostelería y descartan de este modo cualquier perspectiva de educación que les lleve más allá de la enseñanza obligatoria. En la transición a la vida profesional de los jóvenes, las redes de contacto de la comunidad tienen un papel clave. Sin embargo, en sus discursos oficiales la comunidad no deja de animar a los jóvenes a cursar estudios superiores.

2. En la bibliografía psicológica (por ejemplo: Moro, 1998, 2002; Moro, De La Noë y Mouchenik, 2004) y sociológica (Portes y Rumbaut, 2001a y 2001b) se ha advertido de las consecuencias de una inversión de roles entre hijos y padres si los primeros comprenden, desde el punto de vista lingüístico y simbólico, el nuevo entorno mucho mejor que sus padres. En estos casos, los hijos se convierten en padres de sus propios padres, se liberan prematuramente del control parental y se quedan sin guía familiar en el proceso de hacerse adultos en una sociedad nueva. Se da entonces una aculturación disonante entre padres e hijos. En cambio, la aculturación selectiva de Portes y Rumbaut (2001a) requiere por parte de padres e hijos un aprendizaje simultáneo del idioma y de las costumbres del lugar de acogida, así como una inserción simultánea en 
la comunidad para preservar la autoridad parental, evitar demasiados conflictos intergeneracionales y facilitar un bilingüismo fluido entre los hijos.

En la comunidad filipina de Barcelona falla a veces el aprendizaje de los idiomas y de las costumbres por parte de los padres mientras que los hijos los aprenden en sus escuelas, por lo que no se puede hablar de simultaneidad. En estos casos, es la comunidad la que ayuda a reforzar la autoridad de los padres, debido a la inmersión total de los mismos en temas laborales, y es también la encargada de transmitir las culturas y las lenguas del país de origen. Asimismo, ejerce también cierto control sobre los jóvenes, tanto en aspectos académicos como morales. De este modo se evitan las consecuencias negativas de una aculturación disonante entre padres e hijos y los jóvenes filipinos no se quedan sin guías ni referentes, ya que los encuentran en la red comunitaria, aunque su control puede al mismo tiempo coartar a los jóvenes su radio de acción (véase Coleman, 1988). Y mientras que los hijos aprenden los idiomas del lugar de destino en sus escuelas, la comunidad organiza cursos de idiomas del lugar de destino para los adultos.

3 y 4. Los adultos pueden compensar sus limitados recursos económicos y apoyarse mutuamente en la educación de sus hijos, brindando ciertos recursos a los jóvenes. De este modo, el capital social de la comunidad, "lejos de fomentar el encapsulamiento en identidades marginadas y marginales contribuye a favorecer dinámicas de participación social y comunitaria y promueve el desarrollo de mayores niveles de continuidad educativa y de trayectorias académicas de éxito" (Pàmies, 2011, realiza estas observaciones sobre los alumnos de origen marroquí que presentan trayectorias escolares de éxito).

Según el concepto de la aculturación aditiva de Gibson (1988), el éxito o el fracaso escolar de los jóvenes no guarda relación con una mayor o menor aculturación, más bien al contrario: son exitosas las estrategias de adaptación que promueven el éxito académico y la inserción socioeconómica, manteniendo al mismo tiempo los referentes culturales compartidos por los padres y la comunidad (véase Pàmies, 2011).

Gracias al capital social de la comunidad, los jóvenes pueden afrontar también posibles discriminaciones raciales con el apoyo de sus familias o de la comunidad y se les anima para que avancen en sus escuelas y asciendan laboralmente en comparación con la situación sus padres. Gracias a haber integrado estos mensajes, se supone que saben ponderar y rechazar las influencias y encuentros con las subculturas locales o "ravalencas" (en el caso de Barcelona) y evitar así una asimilación descendente.

Asimismo, les ayuda el hecho de tener una religión que comparten con algunos lugareños y que a nivel institucional ha facilitado mucho el trabajo comunitario. Además, al éxito de estos jóvenes contribuye el haber sido educados en los conceptos clave de las culturas filipinas como el pakikisama y la hiya, así como el afán por las smooth interpersonal relationships, el respeto a los mayores y la disciplina, que les llevan a tener aversión a las conductas conflictivas y les facilitan seguir un comportamiento correcto, "silencioso", que puede disminuir la propensión a crear

\footnotetext{
${ }^{11}$ Así describió una enfermera a sus pacientes filipinos en el Raval.
} 
conflictos sociales en el espacio público. Todos ellos son atributos que, conjuntamente con la supervaloración de la educación escolar, convierten a los niños y a los adolescentes filipinos en alumnos muy valorados por sus profesores.

Según los criterios que favorecen el éxito académico de jóvenes migrantes elaborados por Suárez Orozco y Suárez Orozco (2008) y otros identificados durante nuestro estudio de campo se puede resumir los factores siguientes respecto a la comunidad filipina:

\begin{tabular}{|c|c|}
\hline FACTORES POSITIVOS & COMUNIDAD FILIPINA EN BARCELONA \\
\hline $\begin{array}{l}\text { Casas/familias estables, sin demasiados } \\
\text { conflictos }\end{array}$ & $\begin{array}{l}\text { Parece que a menudo sí pero no de manera } \\
\text { generalizada }\end{array}$ \\
\hline Haber llegado con toda la familia & Muchas veces no \\
\hline $\begin{array}{l}\text { Padres se implican en la educación, motivan, } \\
\text { apoyan }\end{array}$ & $\begin{array}{l}\text { Sí, con bastante insistencia aunque a veces no } \\
\text { pueden por sus horarios }\end{array}$ \\
\hline $\begin{array}{l}\text { Padres tienen recursos para controlar los } \\
\text { procesos educativos }\end{array}$ & Muchos sí \\
\hline Recursos en la comunidad & Muchísimos, además formalmente organizados \\
\hline Escuelas relativamente/poco/nada problemáticas & Sí, las escogen en función de esto \\
\hline Profesores/mentores/tutores que apoyan, motivan & $\begin{array}{l}\text { Sí, les gusta trabajar con sus alumnos filipinos. } \\
\text { Algunos incluso les dan clases extra gratuitas }\end{array}$ \\
\hline Dominio castellano/catalán & Entre los reagrupados a menudo falla \\
\hline $\begin{array}{l}\text { Género (apoyo o represión o obstáculos para la } \\
\text { educación) }\end{array}$ & $\begin{array}{l}\text { Igualdad respecto a la necesidad de una buena } \\
\text { educación, sin embargo a nivel universitario a } \\
\text { veces impiden que las chicas vayan a estudiar fuera } \\
\text { del lugar de los padres (aunque sea a una } \\
\text { institución élite) }\end{array}$ \\
\hline Madres (padres) con un nivel educativo alto & Muchos sí, pero no todos \\
\hline Padres con profesiones de alto nivel social & Casi nadie, trabajos muy humildes \\
\hline $\begin{array}{l}\text { Papeles en orden, sin estrés por estar } \\
\text { indocumentado }\end{array}$ & Casi todos \\
\hline $\begin{array}{l}\text { Sin asuntos traumáticos (exiliados) que hace falta } \\
\text { elaborar }\end{array}$ & Casi nadie \\
\hline $\begin{array}{l}\text { Implicación conductual en la escuela de parte de } \\
\text { los alumnos }\end{array}$ & Muchos, casi todos \\
\hline $\begin{array}{l}\text { "Contextos de salida", "ventajas } \\
\text { premigratorias"12 }\end{array}$ & $\begin{array}{l}\text { Muchos ya llegan con buenos conocimientos en } \\
\text { ciencias naturales, inglés, música y mucha } \\
\text { motivación en deporte. Además con una idea de } \\
\text { esfuerzo y ascenso social interiorizada }\end{array}$ \\
\hline $\begin{array}{l}\text { Transmisión transcultural de la importancia de la } \\
\text { educación }\end{array}$ & Normalmente sí y de manera reiterante \\
\hline
\end{tabular}

Tabla 1: Factores positivos para el éxito académico

Por supuesto, nunca se trata de estereotipar a una comunidad en especial, siempre son más importantes los factores individuales, como también las capacidades y

${ }^{12}$ Suárez Orozco y Suárez Orozco, 2008: 68 y véase también Dronkers (2010). 
talentos, la agencia y resistencia. Pero la tabla debería servir también para poner de manifiesto las capacidades receptoras de la sociedad de acogida y cómo las manifiesta con un colectivo en concreto.

\section{Conclusión}

Por el capital humano de muchos padres y el capital social de la comunidad, los jóvenes filipinos no se han quedado sin guía ni han perdido la relación con sus culturas de origen. A la mayoría les transmiten constantemente la importancia de la educación, apoyándoles en sus estudios $\mathrm{y}$, a veces, presionándoles, y se insiste en el éxito académico de manera transcultural entre Filipinas, Barcelona y otros destinos de la diáspora filipina. El capital social se manifiesta aquí en la reciprocidad y la capacidad acumulativa de la comunidad para cooperar y trabajar juntos por el bien de todos, que se manifiesta en la educación como instrumento para el ascenso social de los jóvenes y para el aumento de su capital humano. Sus éxitos serían los puestos de trabajo valorados, es decir, son convertibles en capital financiero (Añonuevo y Añonuevo, 2002; Coleman, 1988). La mutualidad y la solidaridad ya están ancladas en las culturas filipinas, a su vez ubicadas entre el individualismo y el comunitarismo (Izquierdo, 2007; Aguilar, 2009), por los conceptos del pakikipagkapwa (orientación de las acciones hacia el prójimo), pakikisama, bayanihan y del utang na loob (la buena convivencia que incluye u obliga a los favores mutuos, a veces más allá de los propios medios económicos). De este modo la funcionalidad y utilidad del capital social descritas por Coleman (1988) en términos más individualistas tienen que ser complementadas por los aspectos del comunitarismo que lleva a una clara consideración del capital social para las acciones sociales, más allá de los intereses contabilizables según parámetros inmediatos, monetarios e individuales. Los beneficios resultan de la pertenencia a un grupo que a su vez brinda una solidaridad que se consigue mediante un trabajo continuo de mantenimiento de los lazos (Bourdieu, 1980).

También, la creación y el mantenimiento del capital social de la comunidad filipina en Barcelona y en otros lugares de la diáspora se considera como una respuesta a la mercantilización total de los cuerpos promovida por los gobernantes de Filipinas en congruencia con las dinámicas políticas y económicas del Norte Global.

De todos modos, hay una "corresponsabilidad educativa", entre la comunidad, las familias y las escuelas (Benhammou y Argemí, 2012: 50) y los rendimientos escolares son buenos, al menos satisfactorios, y, en algunos casos, sobresalientes. Los jóvenes suelen incorporarse a los ciclos formativos o a las universidades. La primera opción es escogida para poder insertarse más rápidamente en el mundo laboral o también para aprovechar el tiempo mientras se mejoran los conocimientos lingüísticos, con la finalidad de acceder después a la universidad con una materia relacionada con la formación profesional realizada (por ejemplo: se hace una formación como auxiliar de enfermería para después estudiar enfermería en la universidad). Solo unos pocos terminan con la enseñanza obligatoria secundaria para insertarse directamente en el mundo laboral de los servicios domésticos o de la hostelería. Cabe esperar algunos 
años para ver si los graduados logran una exitosa inserción en el mercado laboral catalán pero ya ahora se puede indicar que los rendimientos académicos de los jóvenes de origen filipino no coinciden con el promedio de jóvenes inmigrantes, que están en una situación de desventaja educativa en comparación con los alumnos autóctonos (Ferrer, Castel y Valiente, 2009; Marí-Klose et al., 2009) y de subrepresentación en las universidades, debido a un previo abandono de la educación postobligatoria, condición que les lleva a unas posibilidades laborales precarias (Feixa et al., 2010 y para los jóvenes de origen marroquí: Pàmies, 2011; Carrasco et al., 2009, con más referencias bibliográficas).

\section{Referencias bibliográficas}

AGUILAR, F.V.Jr. (2009). Maalwang Buhay: family, overseas migration, and cultures of relatedness in Barangay Paraiso. [Con John Estanley Z. Penalosa, Tania Belen T. Liwanag, Resto S. Cruz y Jimmy M. Melendrez]. Quezon City: Ateneo de Manila University Press.

AÑONUEVO, E. y AÑONUEVO, A.T. (Eds.). (2002). Coming Home. Women, Migration and Reintegration. Manila: Balikbayani Foundation, Inc y Atikha Overseas Workers and Communities Initiatives, Inc.

APARICIO, R.; PORTES, A. y HALLER, W. (2009). La Segunda Generación en Barcelona: Un Estudio Longitudinal. Primer informe. Madrid: Universidad Comillas.

ASIS, M.M.B. (2008). The Social Dimensions of International Migration in the Philippines. Findings from Research. En M.M.B. Asis y F. Baggio (Eds.). Moving Out, Back and Up: International Migration and Development Prospects in the Philippines (pp. 77-108). Quezon City: Scalabrini Migration Center.

BAGGIO, F. (2008). The Migration-Development Disconnect in the Philippines. En M.M.B. Asis y F. Baggio (Eds.). Moving Out, Back and Up: International Migration and Development Prospects in the Philippines (pp. 110-126). Quezon City: Scalabrini Migration Center.

BAGGIO, F. (2009). Voices of Filipino Migrants in Italy and Spain. Executive Summary of Findings of the MAPID Research Activities in Italy. En MAPID Migrants' Associations and Philippine Institutions for Development. Capacity Building Programs in the Philippines, Italy and Spain. Reference Materials (pp. 122). Disponible en [CD-ROM]. Quezon City: Scalabrini Migration Center.

BELLO, W. (2004). The Anti-Development State: The Political Economy of Permanent Crisis in the Philippines. Londres y Nueva York: Zed Books.

BENHAMMOU, F. y ARGEMÍ, R. (2012). El papel esencial de la educación. Cuadernos de pedagogía, 420, 48-50.

BOURDIEU, P. (1980). Le capital social. Notes provisoires. Actes de la recherche en sciences sociales, 31, 2-3. 
CARRASCO, S.; PÀMIES, J. y BELTRAN, M. (2009). Familias inmigrantes y escuela: desencuentros, estrategias y capital. Revista Complutense de Educación, 20, núm. 1, 1-28.

COLEMAN, J. (1988). Social Capital in the Creation of Human Capital. American Journal of Sociology, 94, 95-119.

DRONKERS, J. (2010). Influència dels països d'origen i de destí en el rendiment de l'alumnat d'origen immigrat. Texto de la conferencia de Jaap Dronkers en el Auditori MACBA de Barcelona el 9 de febrero de 2010 en el marco de Debats d'Educació, Fundació Bofill.

FEIXA, C.; ROMANÍ, O.; HALIM, N.; LATORRE REOLON, A.; PORZIO, L. y RODRÍGUEZ, A. (2010). Spain: Irregular Lives in the Southern Rim of Europe. En K. Fangen, K. Fossan y F. A. Mohn (Eds.). Inclusion and Exclusion of Young Adult Migrants in Europe. Barriers and Bridges (pp. 17-49). Farnham y Burlington: Ashgate.

FERRER, F. (Dir); CASTEL, J.L. y VALIENTE, O. (2009). Equitat, excel-lència $i$ eficiència educativa a Catalunya. Una anàlisi comparada. Col. Polítiques $n^{\circ} 68$. Barcelona: Fundació Jaume Bofill.

GÁNDARA, P. y CONTRERAS, F. (2010). The Latino education crisis: The consequences of failed social policies. Cambridge: Harvard University Press.

GIBSON, M. (1988). Accommodation without Assimilation. Sikh immigrants in an American High School. Nueva York: Cornell University Press.

GUTIÉRREZ-OTERO, A. (2007). La comunitat filipina a Barcelona. La seva importància en la vida dels i les immigrants filipins. En M.J. Izquierdo (Dir.). Servidores sense fronteras. La migració femenina filipina (pp. 41-63). Publicacions Digitals, núm. 3. Disponible en [CD-ROM]. Barcelona: Fundació Bofill.

HSIA, J. (1988). Asian Americans in Higher Education and at Work. Hillsdale: Erlbaum.

HUNE, S. y CHAN, K. S. (1997). Special Focus: Asian Pacific American Demographic and Educational Trends. En D. J. Carter y R. Wilson (Eds.). Fifteenth Annual Status Report on Minorities in Higher Education, 1996-1997. Washington, D.C.: American Council on Education, 1997.

IZQUIERDO, M.J. (Dir.). (2007). Servidores sense fronteras. La migració femenina filipina. Publicacions Digitals, núm. 3. Disponible en [CD-ROM]. Barcelona: Fundació Bofill.

JULIANO, D. (2003). La enseñanza de las Ciencias Sociales, Geografía e Historia desde un punto de vista no androcéntrico. Estado de cuestión y líneas de avance. En E. Hidalgo, D. Juliano, M. Roset y A. Caba. Repensar la enseñanza de la geografía $y$ de la historia. Una mirada desde el género (pp. 17-26). Barcelona: Octaedro.

LE ESPIRITU, Y. y WOLF, D.L. (2001). The Paradox of Assimilation: Children of Filipino Immigrants in San Diego. En A. Portes y R.G. Rumbaut (Eds.). Ethnicities. 
Children of Immigrants in America (pp. 157-186). Berkeley y Los Angeles: University of California Press/ Nueva York: Russell Sage Foundation.

MARÍ-KLOSE, P.; MARÍ-KLOSE, M., GRANADOS, F.J.; GÓMEZ-GRANELL, C. y Martínez, A. (2009). Informe de la inclusión social en España. Barcelona: Fundació Caixa Catalunya.

MARXEN, E. (2009). La etnografía desde el arte. Definiciones, bases teóricas y nuevos escenarios. Alteridades, 19 (37), 7-22.

MORO, M.R. (1998). Psychothérapie transculturelle des enfants de migrants. Paris: Dunod.

MORO, M.R. (2002). Enfants d'ici venus d'ailleurs. Paris: La Decouverte.

MORO, M.R., DE LA NOË, Q. y MOUCHENIK, Y. (2004). Manuel de psychiatrie tranculturelle. Travail clinique, travail social. Grenoble: La Pensée Sauvage.

PAMIES, J. (2011). Éxito académico, inmigración y ciudadanía. Condiciones y posibilidades entre jóvenes de origen marroquí en Cataluña. En F.J. García Castaño y N. Kressova. (Coords.). Actas del I Congreso Internacional sobre Migraciones en Andalucia (pp. 443-448). Granada: Instituto de Migraciones.

PARREÑAS SALAZAR, R. (1998). The Global Servants: (Im)Migrant Filipina Domestic Workers in Rome and Los Angeles. Tesis doctoral. University of California, Berkeley.

PARREÑAS SALAZAR, R. (2001). Servants of Globalization: Women, Migration and Domestic Work. Stanford: Stanford University Press.

PARREÑAS SALAZAR, R. (2006). Children of Global Migration: Transnational Families and Gendered Woes. Quezon City: Ateneo de Manila University Press.

PINGOL, A. (2007). Cura i globalització: una visió filipina. En M.J. Izquierdo (Dir.). Servidores sense fronteras. La migració femenina filipina (pp. 90-110). Publicacions Digitals, núm. 3. Disponible en [CD-ROM]. Barcelona: Fundació Bofill.

PORTES, A. y RUMBAUT, R.G. (2001a). Legacies. The Story of the Immigrant Second Generation. Berkeley y Los Angeles: University of California Press/ Nueva York: Russell Sage Foundation.

PORTES, A. y RUMBAUT, R.G. (Eds.). (2001b). Ethnicities. Children of Immigrants in America. Berkeley y Los Angeles: University of California Press/ Nueva York: Russell Sage Foundation.

RIBAS, N. (1994). Origen del proceso emigratorio de la mujer filipina a Cataluña. Papers de Sociologia, 43, 101-114.

RIBAS, N. (2004/2005). La feminización de las migraciones desde una perspectiva filipina. Revista CIDOB d'Afers Internacionals, 68, 67-87.

RUMBAUT, R. (1997). Ties that Bind: Immigration and Immigrant Families in the United States. En A. Booth, A. C. Crouter y N. Landale (Eds.). Immigration and the 
Family: Research and Policy on U.S. Immigrants (pp. 3-46). Mahwah: Lawrence Erlbaum Associates.

SCALABRINI MIGRATION CENTER (PRODUCTOR) y BAGGIO, F. (DIRECTOR). (2006). Chasing Rainbows. Young Pinoys Ponder the Future [DVD]. Quezon City: Scalabrini Migration Center.

SERRA, C. y PALAUDÀRIES, J. M. (2010). Continuar o abandonar. L'alumnat estranger a l'educació secundària. Barcelona: Fundació Jaume Bofill.

SUÁREZ-OROZCO, M.; SUÁREZ-OROZCO, C. y TODOROVA, I. (2008). Learning a new land: immigrant students in American Society. Cambridge: Harvard University Press.

SUÁREZ-OROZCO, M. y SUÁREZ-OROZCO, C. (2008). Históries d'immigració: la comprensió dels patrons de rendiment escolar dels joves immigrants nouvinguts. Barcelona: Fundació Jaume Bofill.

SUBIRATS, J. y RIUS, J. (Dir.). (2006). Del Chino al Raval. Cultura y transformación en la Barcelona central. Barcelona: Centre de Cultura Contemporànea de Barcelona.

SUZUKI, B. (2002). Revisiting the Model Minority Stereotype: Implications for Student Affairs Practice and Higher Education. New Directions for Student Services, 97, 21-32.

VILLARROYA, E. (2010). Filipino Migrants' Associations in Spain as Potential Agents of Change. En F. Baggio (Ed.). BRICK by BRICK. Building Cooperation between the Philippines and Migrants' Associations in Italy and Spain (pp. 255336). Quezon City: Scalabrini Migration Center.

WOLF, D.L. (1997). Family Secrets: Transnational struggles among children of Filipino immigrants. Sociological Perspectives, 40, 455-482.

WONG, P.; FAITH LAI, C.; NAGASAWA, R. y LIN, T. (1998). Asian Americans as a model minority: self-perceptions and perceptions by other racial groups. Sociological Perspectives, 41, 95-118.

ZANFRINI, L. y ASIS, M.M.B. (a cura di). (2006). Orgoglio e pergiudizi. Una ricerca tra Filippine e Italia sulla transizione all'età attiva dei figli di emigranti e dei figli di immigranti. Milán: Franco Angeli.

\section{Correspondencia con la autora:}

Eva MARXEN

c/ Cendra 14, 1

08001 Barcelona

espai_dart@yahoo.es

www.evamarxen.com 